Check for updates

Cite this: RSC Adv., 2017, 7, 20900

\title{
Bioactive titaminates from molecular layer deposition $\uparrow$
}

\begin{abstract}
L. Momtazi, (D) ${ }^{a}$ H. H. Sønsteby, ${ }^{a}$ D. A. Dartt, ${ }^{b}$ J. R. Eidet ${ }^{c}$ and O. Nilsen ${ }^{\star a}$
Organic-inorganic hybrid materials are an emerging class of materials suitable for deposition by the molecular layer deposition (MLD) technique. Their toolbox is now expanded to include linkers of amino acids, which when combined with titanium form materials that can be termed titaminates, based on the amine and carboxylates present in the amino acids being used as linkers. This is a class of compounds with high potential as bioactive materials, containing essential amino acids and biocompatible titanium. The films have been prepared by combining titanium tetra-isopropoxide (TTIP) with glycine and L-aspartic acid. L-Arginine has also been used, however, without success. Hybrid films of TTIP and succinic acid were also investigated as a comparison to L-aspartic acid due to their structural similarities. All systems show self-limiting growth with a reduction in the growth rate with increasing temperature. The as-deposited films are amorphous, have low surface roughness, exhibit a hydrophilic nature as measured by a goniometer, and bear indications of some porosity towards water. Films based on glycine and L-aspartic acid have been used as substrates for growth of epithelial cells (rat goblet cells) where their proliferation has been monitored. The cell proliferation was significantly increased on these substrates compared to uncoated coverslips.
\end{abstract}

Received 15th February 2017

Accepted 30th March 2017

DOI: 10.1039/c7ra01918a

rsc.li/rsc-advances molecules, such as amino acids. The precursors are introduced onto a substrate in a sequential manner separated by purging steps to remove unreacted precursors. By doing this, gas phase reactions are avoided and uniform coverage even on complex three-dimensional substrates can be achieved. The cyclic nature of the process also enables monolayer control of the deposited films and provides the possibility to vary the type of building units as the film grows, ensuring control on the molecular level..$^{4-8}$ Like ALD, MLD requires precursors with sufficient vapor pressure, reactivity and stability against decomposition at sublimation and deposition temperatures. ${ }^{3}$ This can be challenging when amino acids are applied as precursors, however, still possible, as will be shown in this paper.

ALD was primarily designed for deposition of inorganic compounds in the late 1970s. ${ }^{9}$ Since then, the range of applicable precursors by this technique has expanded continuously. Recently, the advancement of the MLD technique enabled formation of hybrid materials by using different types of organic and inorganic precursors. ${ }^{\mathbf{1 0 - 1 2}}$ The major motivation for deposition of hybrid materials by MLD has so far been as gas permeation barriers and for its presumed flexibility. ${ }^{\mathbf{1 3 , 1 4}}$ Moreover, it enables modification of optical and electrical properties of deposited films in a gradual manner. ${ }^{15}$

Titanicones are an example of hybrid materials prepared by the MLD technique constructed using functional alcohols and titanium precursors. Titanicones have possible applications in fabricating flexible multilayer gas diffusion barriers and thin solar films. ${ }^{13}$ We here introduce a new novel group of organic-
N-0315 Oslo, Norway. E-mail: ola.nilsen@kjemi.uio.no

${ }^{b}$ Schepens Eye Research Institute, Massachusetts Eye and Ear, Department of Ophthalmology, Harvard Medical School, 20 Staniford St, Boston, MA, USA

'Oslo University Hospital, Department of Ophthalmology, Kirkeveien 166, 0407 Oslo, Norway

$\dagger$ Electronic supplementary information (ESI) available. See DOI: 10.1039/c7ra01918a 
inorganic hybrid material that we have termed titaminates, based on the amine and carboxylates present in the amino acids used as linkers.

Design of bioinert and bioactive surfaces is a huge field where ALD/MLD has not yet been extensively applied. In medical applications, especially for bone implants, surfaces that do not cause inflammatory reactions can form stable connective tissue around the implant and facilitate healing processes. The most important factors in designing for biocompatibility is by control of the surface wetting properties of the film, in addition to surface charge, type of functional groups, and surface topography. ${ }^{\mathbf{1 6}}$

Cell-surface interactions are mediated through specific proteins that bind to specific amino acid sequences in an extracellular matrix in our body, such as RGD. ${ }^{16}$ RGD is a tripeptide composed of the three amino acids; L-arginine, glycine and L-aspartic acid (Fig. 1). Because RGD is proven to be highly effective at promoting the attachment of numerous cell types to a plethora of diverse materials, these three amino acids were selected as the organic building blocks for our MLD processes. ${ }^{17}$

Among inorganic materials, titanium-based coatings are recognized as compounds that support bone forming cells due to protein absorption and platelet activation with subsequent release of growth factors. ${ }^{\mathbf{1 6}}$ Titanium is considered one of the most biocompatible elements ${ }^{\mathbf{1 8}}$ suitable as basis for MLD growth.

In this study, we expand the MLD toolbox to include essential $\alpha$-amino acids using titanium as the metal center. We have chosen titanium tetra-isopropoxide (TTIP) as the titanium source, avoiding the corrosive $\mathrm{HCl}$ by-product formed when using the more available and reactive $\mathrm{TiCl}_{4}$ precursor. Lowtemperature deposition using TTIP is prone to some carbon contamination in the resulting films, but this is not considered to be a problem for the current applications. This work is a contribution towards development and characterization of a novel type of hybrid materials with potential applications as bioactive surfaces. In addition, due to the structural similarity between aspartic acid and succinic acid (Fig. 1), hybrid films of Ti-succinic acid were also deposited for comparison. This should reveal effects of the additional amine group on bonding principles and steric hindrance between organic precursor and TTIP on the film growth rate.

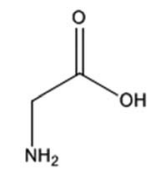

Glycine

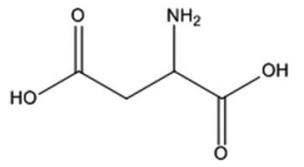

L-aspartic acid

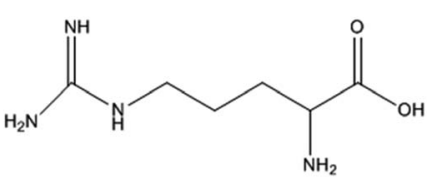

Arginine

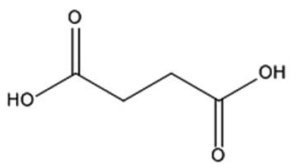

Succinic acid
Fig. 1 The organic molecules used as precursors in the current study.
The bioactivity of a selection of these hybrid films was studied by measuring the proliferation of an epithelial cell, rat goblet cells on glass coverslips with these novel coatings. Goblet cells in the conjunctiva are one of the main cell types that cover the ocular surface and their main function is to produce mucins that support a stable and functional tear film. Any disturbance in the tear film components can cause ocular surface diseases such as dry eye disease. ${ }^{19}$ The proliferation rate of an epithelial cell, rat goblet cells is known to be sensitive to the substrate, and is hence a suitable marker for investigation of bioactivity of different surfaces.

\section{Experimental}

The films were deposited in an F120-Sat reactor (ASM Microchemistry Ltd) using TTIP and glycine, L-arginine, L-aspartic acid and succinic acid as precursors. The different organic precursors are sketched in Fig. 1 together with their names as used in this paper. Nitrogen was used as carrier gas supplied at a total rate of $500 \mathrm{~cm}^{3} \mathrm{~min}^{-1}$ from a Schmidelin-Sirocco-5 $\mathrm{N}_{2}$ generator with a purity of $99.999 \%$ with respect to $\mathrm{N}_{2}$ and $\mathrm{Ar}$ content. The films were deposited on precleaned single crystal substrates cut from $\mathrm{Si}(100)$ wafers.

The growth rate was measured as a function of deposition temperature for each TTIP + organic precursor pair. In total, the temperature range of $160-375{ }^{\circ} \mathrm{C}$ was covered. Thickness and refractive index of the films were measured by a J. A. Woollam alpha-SE spectroscopic ellipsometer at incident angle of $70^{\circ}$. The films were assumed transparent and the data were fitted using a Cauchy-model.

Infrared spectra of the films was obtained with a Fourier transform infrared (FTIR) transmission spectroscopy using a Bruker VERTEX 80 FTIR spectrometer. The instrument was equipped with a nitrogen purging system. An uncoated $\mathrm{Si}(100)$ substrate was used to collect the background.

X-ray photoelectron spectroscopy (XPS) was performed using a Thermo Scientific Theta Probe Angle-Resolved XPS system. The energy was charge referenced to adventitious C 1s, C-C peak, at $284.8 \mathrm{eV}$. The instrument was equipped with a standard $\mathrm{Al} \mathrm{K} \alpha$ source $(h \nu=1486.6 \mathrm{eV})$, and the analysis chamber pressure was in the order of $10^{-8}$ mbar. Pass energy values of 200 and $50 \mathrm{eV}$ were used for survey spectra and detailed peak scans, respectively. Ti 2p, C 1s and $\mathrm{O}$ 1s were captured for all samples and $\mathrm{N}$ 1s was captured for $\mathrm{L}$-aspartic acid and glycine.

The density of the films was measured by a Bruker AXS D8 advance film diffractometer equipped with a LynxEye strip detector. The thin film diffractometer had a Göbel mirror and a $\mathrm{Ge}(220)$ four bounce monochromator for X-ray reflectivity (XRR) measurements.

Atomic force microscopy (AFM) measurements were performed in noncontact mode using a Park XE70 and contact angle measurements were done by a ramé-hart contact angle goniometer and DROP image analysis program.

The growth dynamics were monitored in situ by a quartz microbalance (QCM) using a Maxtek TM400 unit and a homemade crystal holder. The changes in resonance frequency of the crystals were linearly dependent on the mass of deposited film, 
which was in accordance with the Sauerbrey equation. ${ }^{20}$ The QCM data were further processes to increase statistics by averaging 16 consecutive cycles. The conversion from frequency to mass per area was performed by using internal standards throughout the deposition campaign of a material with known growth rate and density, as measured by XRR. In this manner, variations in surface area of the QCM crystal due to evolution of texture was calibrated.

The bioactivity of the surfaces was studied using conjunctival tissue that was removed from both eyes of male SpragueDawley rats. All removal of tissue and subsequent manipulations in this study conformed to the guidelines established by the ARVO Statement for the Use of Animals in Ophthalmic and Vision Research and was approved by the Schepens Eye Research Institute Animal Care and Use Committee. Pieces of minced conjunctiva (explants) were cultured in RPMI 1640 medium (BioWhittaker, Walkersville, MD) supplemented with $10 \%$ fetal bovine serum (FBS) (HyClone Laboratories, Logan, UT), $2 \mathrm{mM}$ glutamine (Lonza, Walkersville, MD), and $100 \mathrm{mg}$ $\mathrm{ml}^{-1}$ penicillin-streptomycin (BioWhittaker). Goblet cells were allowed to grow out from the explant for one week, after which the explant was removed from the culture. The grown goblet cells were then detached by trypsin and sub-cultured for 4 days on amino acid coated substrates in the culture medium described above.

To quantify the amount of viable cells in culture after 4 days, which is an indication of cell proliferation and survival, the alamarBlue ${ }^{\circledR}$ (Invitrogen) colorimetric indicator was used. This is a nontoxic reagent that does not affect cell viability or proliferation. The active component in alamarBlue, the nonfluorescent resazurin, is reduced to the fluorescent resorufin inside viable cells. Thus, this assay measures the amount of live cells in culture. The goblet cells were seeded on the substrates in 24-well plates. The cells were incubated in culture medium with $10 \%(\mathrm{v} / \mathrm{v})$ alamarBlue solution for 4 hours before the cultures had become completely confluent. After incubation, the culture medium was transferred to 96-well plates, and the amount of fluorescence from resorufin was measured at $560 \mathrm{~nm}$ excitation and $590 \mathrm{~nm}$ emission wavelength, using the Synergy Mx fluorescence plate reader (BioTek). Three independent experiments were done in triplicate.

\section{Results}

The sublimation temperature of the organic precursors was initially tested in a home built unit where the temperature of a small amount of the precursor was increased at a rate $c a .2{ }^{\circ} \mathrm{C}$ per minute under vacuum while recording any visual change by a camera and any deposition on a nearby QCM sensor by logging its resonance frequency. The results from this test are given in Table 1 together with the values proved suitable for the later MLD depositions.

The visual imaging of the precursors showed complete sublimation for all of the precursors except for arginine. Although the QCM analysis showed an abrupt increase in mass at $200{ }^{\circ} \mathrm{C}$ for arginine, the precursor melted and gave signs indicating thermal decomposition. Further XPS analysis of films based on arginine showed presence of nitrogen in the film. However, the low thermal stability of this precursor raised questions about whether the nitrogen in the film originated from $\mathrm{L}$-arginine itself or from thermal decomposition products of this precursor. Arginine was therefore not used in the further experiments due to uncertainty related to its stability.

The growth dynamics for MLD of the individual systems were investigated by QCM to obtain information on suitable pulse and purge parameters. An overview of the growth dynamics for long pulses and purges is given in Fig. 2 and the results from a systematic variation of the pulse and purge times are given in ESI 1-3. $\dagger$ The deposition conditions and the obtained pulse and purge times extracted from this investigation are given in Table 1. All three investigated systems follow near self-limiting growth where excess precursors and by-products are removed during the following purge step.

The growth rate as a function of deposition temperature of the individual systems (Fig. 3) was further mapped based on the pulsing parameters given in Table 1 . Glycine and L-aspartic acid showed relatively constant, albeit somewhat declining, growth rate over a large temperature range. The growth rate of succinic acid was more dependent on the deposition temperature with a maximum at $180{ }^{\circ} \mathrm{C}$.

The evolution in refractive index as measured by ellipsometry, and density as measured by XRR as a function of deposition temperature, is given in Fig. $4 \mathrm{a}$ and $\mathrm{b}$. The index of refraction increased on an overall basis nearly linearly with deposition temperature from 1.65 at $155{ }^{\circ} \mathrm{C}$ to 2.15 at $375{ }^{\circ} \mathrm{C}$. For comparison, the index of refraction of anatase $\mathrm{TiO}_{2}$ films deposited at $225^{\circ} \mathrm{C}$ is 2.36 .

The density of the films based on glycine and L-aspartic acid was relatively low and almost unaffected by the deposition temperature. The density of the films based on succinic acid increased somewhat with temperature $\left(3.2-3.5 \mathrm{~g} \mathrm{~cm}^{-3}\right)$ and was notably higher than what one would expected considering that the density of anatase $\mathrm{TiO}_{2}$ is $3.78 \mathrm{~g} \mathrm{~cm}^{-3}$. The results from the XRR analysis is given in ESI $4 . \dagger$

Table 1 Deposition conditions for QCM investigation and obtained pulse and purge parameters

\begin{tabular}{|c|c|c|c|c|c|c|}
\hline Glycine & 200 & 225 & 1 & 1 & 2 & 1 \\
\hline Arginine & 200 & 210 & 1 & 1 & 2 & 1 \\
\hline Succinic acid & 140 & 180 & 2 & 1 & 3 & 1 \\
\hline
\end{tabular}




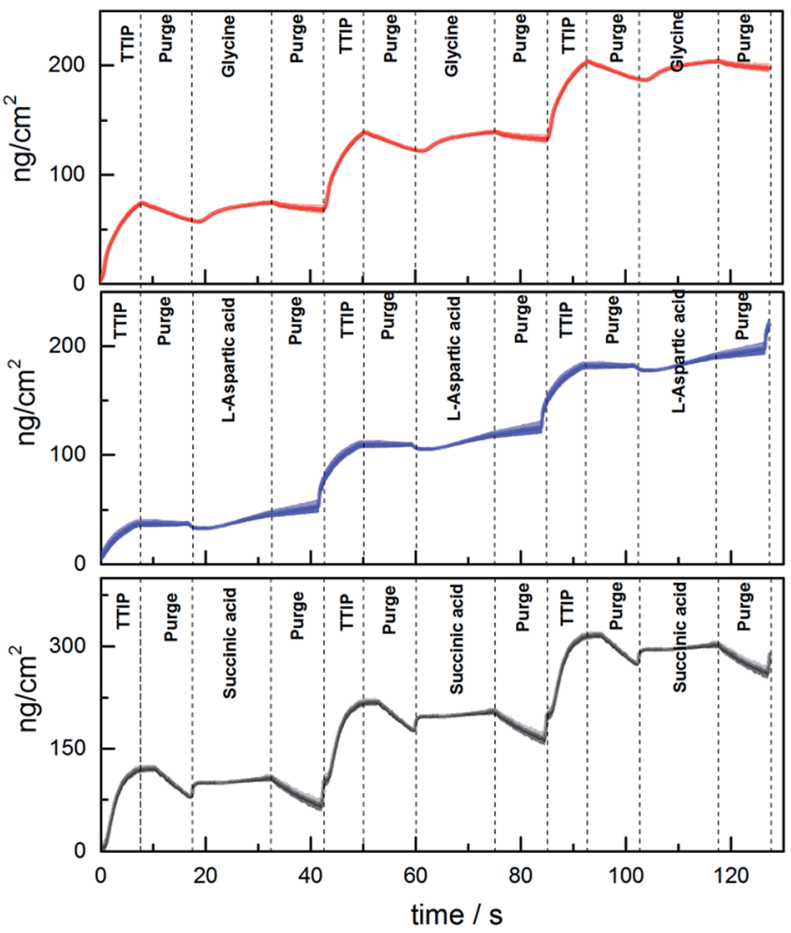

Fig. 2 Mass evolution during growth measured by QCM using TTIP and glycine (at $225^{\circ} \mathrm{C}$ ), L-aspartic acid (at $250^{\circ} \mathrm{C}$ ), or succinic acid (at $180^{\circ} \mathrm{C}$ ). The shaded area represents the statistical variation during 16 cycles.



Fig. 3 Film growth as a function of deposition temperature for TITIP and glycine (red squares), L-aspartic acid (blue triangles) and succinic acid (black circles).

By comparing the growth of the different systems as given by the QCM analysis, it is evident that the mass increase during the TTIP pulse is highest for the succinic-acid system, being the only system without amines. Neither of the systems shows a significant mass increase during pulsing of the organic precursor. This is in accordance with a ligand exchange of the rather heavy isopropanol groups with the organic precursors, giving a low net mass increase.

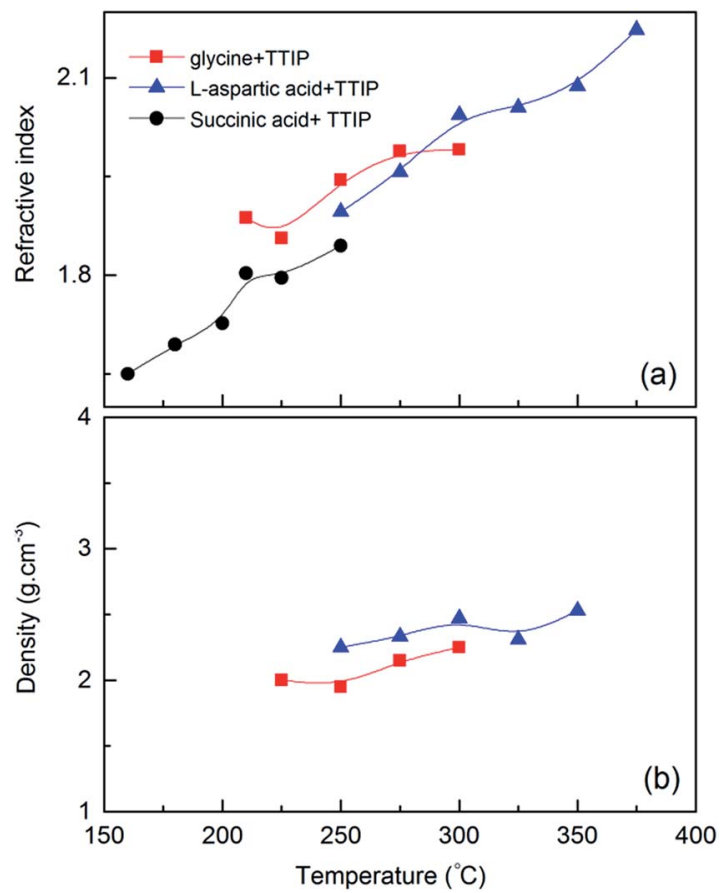

Fig. 4 (a) Refractive index at $632.8 \mathrm{~nm}$ as measured by spectroscopic ellipsometry and (b) film density as measured by XRR as a function of substrate temperature for TTIP and glycine (red squares), L-aspartic acid (blue triangles) and succinic acid (black circles).

FTIR analysis of the hybrid films provide information on the presence of the organic moieties in the films and the resulting bonding modes, Fig. 5. For the amino acids, a weak asymmetric $\mathrm{NH}_{3}{ }^{+}$bending band near $1660-1610 \mathrm{~cm}^{-1}$, and a fairly strong $\mathrm{NH}_{3}{ }^{+}$symmetric bending band near $1550-1485 \mathrm{~cm}^{-1}$ can be detected. The carboxylate group in amino acids absorbs strongly near $1600-1590 \mathrm{~cm}^{-1}$ and weakly near $1400 \mathrm{~cm}^{-1}$, which corresponds respectively to asymmetric and symmetric stretching of $\mathrm{COO}^{-}$. In addition, amino acids show a broad $\mathrm{NH}_{3}{ }^{+}$band in the 3100-2600 region. The absorption bands of carboxylate ions are very close to the absorption bands of $\mathrm{NH}_{3}{ }^{+}$ in amino acids. ${ }^{21,22}$

Carboxylate ions may coordinate to metals in three modes, Fig. 6. The width of the frequency splitting $(\Delta)$ between asymmetric and symmetric stretching band of carboxylic ion determines the type of bonding mode. A splitting in the range 50-150 $\mathrm{cm}^{-1}$ is typical for bidentate complexes, for unidentate complexes $\Delta>200 \mathrm{~cm}^{-1}$ and bridging complexes have $\Delta$ between 130 and $200 \mathrm{~cm}^{-1} .^{23}$

Based on this, the reaction between TTIP and succinic acid appears to be of bidentate type $\left(\Delta \sim 90 \mathrm{~cm}^{-1}\right)$, while the bond type is more uncertain for the amino acid systems since the absorption band for asymmetric carboxylate ion and symmetrical and asymmetrical $\mathrm{NH}_{3}{ }^{+}$is very close. This results in a broad absorption band hampering its analysis. Despite this, the overall appearance favours the interpretation of a larger split supporting bridging complexes.

All three systems show a weak absorption band around 1290 $\mathrm{cm}^{-1}$, which corresponds to C-O stretching of carboxylic acid. ${ }^{24}$ 




Fig. 5 FTIR spectra of hybrid films of (a) amino acid systems with TTIP (b) succinic acid and TTIP for $76 \mathrm{~nm}$ glycine, $53.9 \mathrm{~nm}$ aspartic acid, and $154.6 \mathrm{~nm}$ succinic acid film deposited on $\mathrm{Si}(100)$ substrates at 225,275 , and $190{ }^{\circ} \mathrm{C}$ respectively.

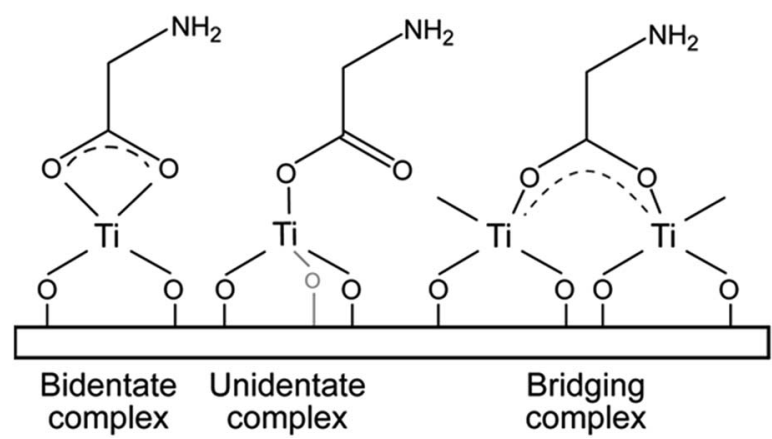

Fig. 6 Possible bonding modes for carboxylic acids towards metals, here represented using glycine.

The broad band in the $3400-2800 \mathrm{~cm}^{-1}$ area is due to presence of $\mathrm{OH}^{-}$groups and can cover the bands from weaker $\mathrm{C}-\mathrm{H}$ and $\mathrm{NH}_{3}{ }^{+}$signals in amino acids.

The $\mathrm{OH}$ stretching mode is absent in FTIR spectra of $\mathrm{L}^{-}$ aspartic acid and succinic acid and very weak and broad for glycine hybrid films. This indicates that almost all $\mathrm{OH}^{-}$groups react with TITIP. The broad absorption band around $3300 \mathrm{~cm}^{-1}$ for glycine film may be due to broad absorption band of $\mathrm{NH}_{3}{ }^{+}$, which is close to $\mathrm{OH}$ absorption band area. It can also result from water absorbed in the film during exposure to air.

Surface topography of films deposited on $\mathrm{Si}(100)$ in temperature range of $200-275{ }^{\circ} \mathrm{C}$ was measured by AFM (Fig. 7). All three systems exhibit very low surface roughness (RMS was $0.3 \mathrm{~nm}, 0.2 \mathrm{~nm}$, and $0.2 \mathrm{~nm}$ for glycine, L-aspartic acid and succinic acid respectively), and almost no distinguishable surface features in spite of the film thicknesses being above $20 \mathrm{~nm}$.

XPS was performed for a qualitative investigation of chemical state in the hybrid thin films based on aspartic acid, glycine and succinic acid, deposited at 250,225 , and $180{ }^{\circ} \mathrm{C}$, respectively. The Ti 2 p core level spectra (Fig. 8) are close to identical for all three sample types. The $459.3 \mathrm{eV}$ binding energy of the $\mathrm{Ti}$ $2 \mathrm{p}_{3 / 2}$-peak corresponds to a Ti-O-type bonding scheme. This is confirmed by the $5.8 \mathrm{eV}$ split spin-orbit energy difference and the charge transfer shake-up satellite corresponding to the $\mathrm{O}$ $2 \mathrm{p}_{\mathrm{eg}} \rightarrow \mathrm{Ti} 3 \mathrm{~d}_{\mathrm{eg}}$ transition. ${ }^{25}$ No evidence of pure Ti-N-bonding is observed, showing that no titanium atoms are only coordinated to nitrogen. The domination of the $\mathrm{Ti}-\mathrm{O}$-character does not, however, rule out Ti-N-bonding.

To observe a possible Ti-N-coordination the $\mathrm{N}$ 1s-peak was captured for aspartic acid and glycine. The $\mathrm{N}$ 1s peak for aspartic acid was sharp and symmetric at a binding energy corresponding to $-\mathrm{NH}_{2}$ only. This points towards hybrid growth on the carboxylic acid group of the organic molecule only. This

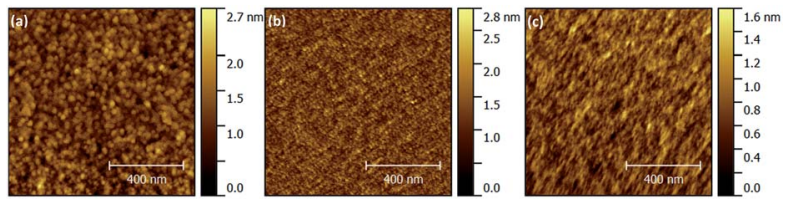

Fig. 7 Surface topography as measured by AFM for films deposited using TTIP and (a) glycine (at $225^{\circ} \mathrm{C}, 41 \mathrm{~nm}$ ), (b) L-aspartic acid (at $275^{\circ} \mathrm{C}, 20 \mathrm{~nm}$ ), (c) succinic acid (at $200{ }^{\circ} \mathrm{C}, 36 \mathrm{~nm}$ ).

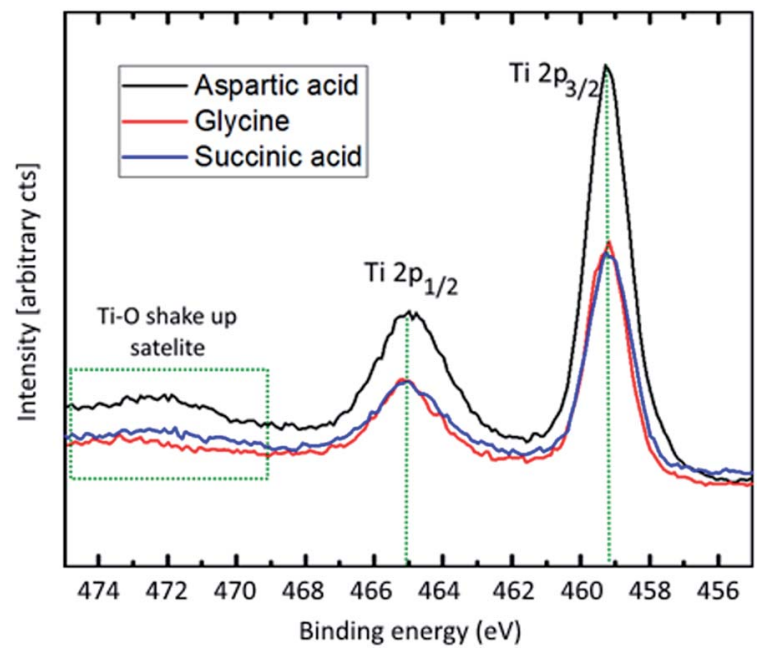

Fig. 8 Ti $2 p$ X-ray photoelectron spectroscopy. Recorded with $50 \mathrm{eV}$ pass energy and monochromatic Al-K $\alpha$-source. 


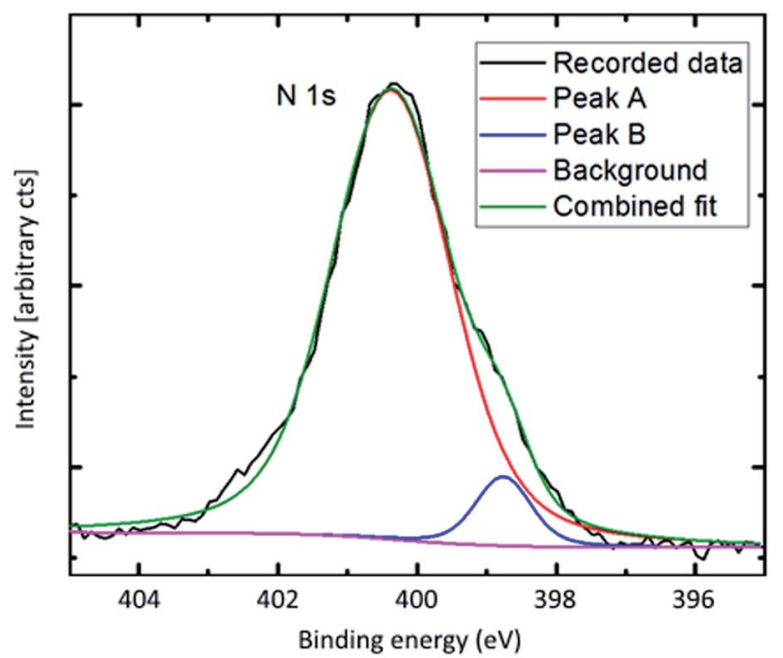

Fig. $9 \mathrm{~N}$ 1s X-ray photoelectron spectroscopy. Recorded with $50 \mathrm{eV}$ pass energy and monochromatic $\mathrm{Al}-\mathrm{K} \alpha$-source.

type of bonding scheme is not as probable for glycine that only has one carboxylic group. The $\mathrm{N}$ 1s peak of the glycine hybrid is not as symmetric, and a second component is needed to fit the total recorded peak (Fig. 9).

This second component has an energy corresponding well with reported energies of O-Ti-N-type bonding, and points towards at least some degree of $\mathrm{O}-\mathrm{Ti}-\mathrm{N}$-linking in the glycine hybrid films. ${ }^{26}$

A quantitative study of the composition of the hybrid films proved difficult due to surface species of carbon and oxygen. However, an approximated $3: 1$ and $2: 1 \mathrm{Ti}: \mathrm{N}$ ratio for aspartic acid and glycine hybrids respectively, can be explained by the as mentioned bonding models. This indicates an excess of $\mathrm{Ti}$ as compared to the ideal stoichiometry. Unfortunately, it was not possible to obtain trustable measurements of the $\mathrm{Ti}$ : O stoichiometry due to oxygen surface species. Attempts at etching the top layer to remove surface species were not successful as the hybrid films were very prone to selective etching.

An important factor that promotes cell attachment is surface wettability. This affects cell growth by determining what type of protein that is adsorbed from solution. The surface wettability depends on surface charge, polarizability and polarity of surface functional groups. Hydrophobic surfaces adsorb albumin, which is an abundant serum protein that does not promote cell attachment. Hydrophilic surfaces, on the other hand, adsorb cell-promoting proteins like fibronectin, which is a component of extracellular matrix (ECM). ${ }^{27}$

The wettability of the surfaces was investigated by measuring the contact angle of water for different films using a goniometer. Hybrid films of glycine and L-aspartic acid were selected for contact angle measurements due to their potential application in cell growth purposes (Fig. 10).

Both films based on glycine and L-aspartic acid were rather hydrophilic with contact angles in the $23-33^{\circ}$ range for films deposited at temperatures in the range $225-350{ }^{\circ} \mathrm{C}$ and did not change significantly with deposition temperature (Fig. 11). This hydrophilicity is highly suitable for cell growth purposes.
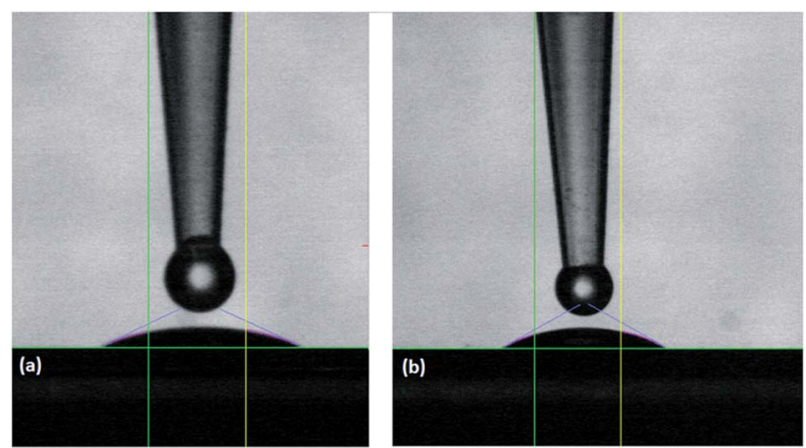

Fig. 10 Contact angle between water and films made of TTIP and (a) glycine (at $250^{\circ} \mathrm{C}$ ), contact angle $=28^{\circ}$ (b) L-aspartic acid (at $275^{\circ} \mathrm{C}$ ) contact angle $=30^{\circ}$.

The growth dynamics of the individual systems were also investigated using an extra water pulse after the organic precursor. The QCM analysis shows a huge mass increase during the water pulse followed by a similar mass loss during its purge, Fig. 12. This indicates that these films can have a spongelike character in the presence of water providing the basis for its high hydrophilicity. Even though the total mass signal increases when water is included, the overall growth rates are reduced some as compared to Fig. 2. The succinic acid system is relatively unchanged from 98 to $91 \mathrm{ng} \mathrm{cm}^{-2}$ cycle, while the L-aspartic and glycine systems change from 72 to $42 \mathrm{ng} \mathrm{cm} \mathrm{cm}^{-2}$

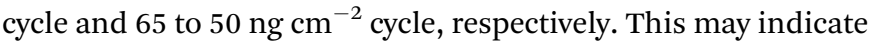
that the relative content of the organic linkers are reduced during growth when water is introduced. Despite this, the idea behind the present test was only meant to shed light on the stability of the hybrid materials during deposition and on its possible porosity.

The cell proliferation and viability results assayed with alamarBlue shows that the amount of viable rat goblet cells on substrates coated with hybrid film of glycine $(p<0.01)$ and aspartic acid $(p<0.01)$ and TTIP is significantly higher than coverslips without any coating (Fig. 13). This indicates that the

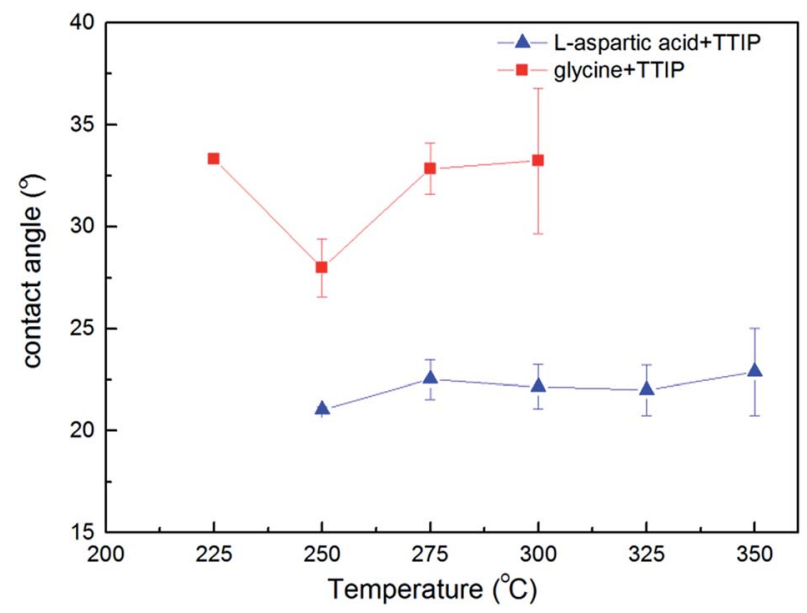

Fig. 11 Contact angle between water and $\mathrm{Ti}-\mathrm{L}$-aspartic acid, and Tiglycine hybrid film versus deposition temperature. 

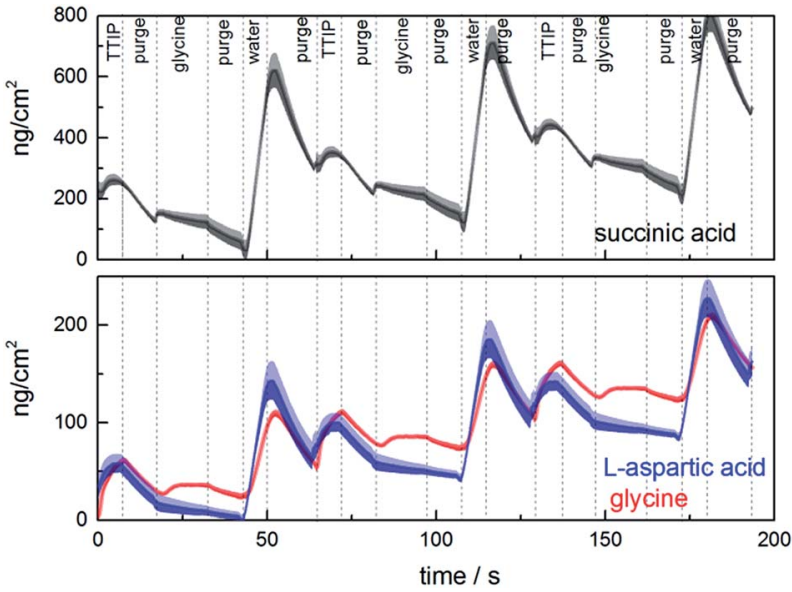

Fig. 12 Mass evolution during growth measured by QCM using TTIP and glycine (at $225^{\circ} \mathrm{C}$ ), L-aspartic acid (at $250^{\circ} \mathrm{C}$ ), or succinic acid (at $180^{\circ} \mathrm{C}$ ), and water. The shaded area represents the statistical variation during 16 cycles.

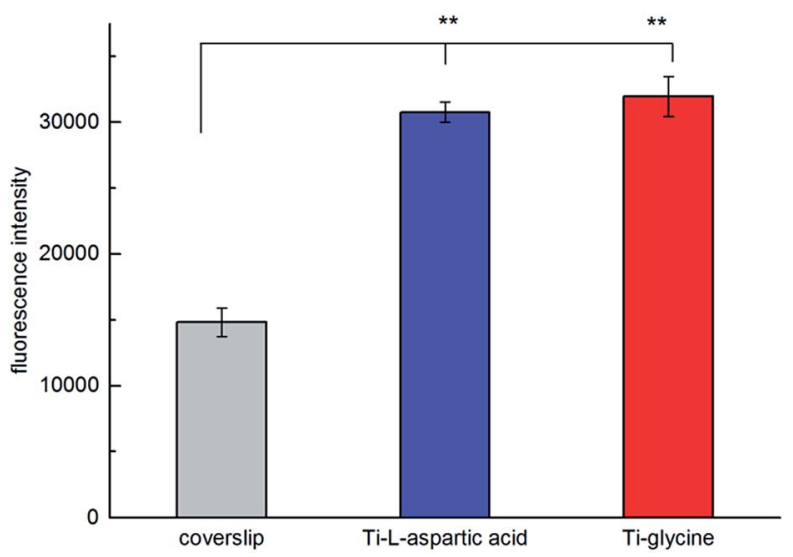

Fig. 13 The effect of hybrid film of Ti-glycine and $\mathrm{Ti}-\mathrm{L}$-aspartic acid on cell proliferation of rat goblet cells as evaluated with alamarBlue assay. ${ }^{*} P<0.01$, compared to coverslip $(n=3)$.

films containing amino acids are highly biocompatible and suitable as scaffolds for cell growth.

\section{Discussion}

The three deposition systems show relatively similar growth dynamics with mass increase during pulsing of TTIP, Fig. 2. However, the aspartic acid system deviates somewhat in that the drop in mass during purging is less noted and that there is an initial decrease in mass during reaction with aspartic acid, where both glycine and succinic acid show an increase in mass. This drop in mass is not reproduced in the short pulse experiments in ESI $2 . \dagger$ It should be noted that the short pulse experiments reports the overall growth rate of a complete cycle, whereas Fig. 2 provides the in situ apparent variation in mass.

When comparing the growth of aspartic acid and succinic acid, the only difference is the presence of an additional amino group for the former. It is evident that this additional functionality reduces the overall growth rate as compared to the succinic acid system. This is despite the observation from XPS analysis that the amino group does not take direct part in the bonding with titanium. Hence, we assume that the additional amino group leads to a lower packing density of aspartic acid on the surface than as for succinic acid. This may also be one possible reason as to why we observe a reduction in mass for pulsing of the aspartic acid, while succinic acid provides an increase in mass. The overall change in mass during exposure of the organic ligand is a sum of the mass lost from reacted isopropanol-ligands and mass gained from the organic ligand. For aspartic acid, it appears that more than two isopropanol groups must desorb for each aspartic acid reacted to obtain an overall reduction in mass. Whereas, the number is smaller for both glycine and succinic acid, leaving the possibility for inclusion of unreacted isopropyl groups in the film during growth. This may be one reason for the differences in overall growth rate, and a slow release of excess isopropanol may explain the loss in mass during both purging sequences. The great number of possible reaction paths and influence of thermal effects makes it challenging to conclude on the growth dynamics and rather highlights the necessity for more thorough investigations.

The growth rates for all the investigated systems decreases with increasing the deposition temperature. According to a recent study, the growth rate of TTIP and water is independent of deposition temperature between 190 and $240{ }^{\circ} \mathrm{C}$ - its ALD window. The ALD window is in fact a combination of precursor reactions and decomposition rates for TTIP. At elevated temperatures pyrolytic decomposition of TTIP becomes dominant and increases the achievable growth rate. ${ }^{28}$ However, as can be seen in Fig. 3, at temperatures higher that $250{ }^{\circ} \mathrm{C}$ the growth rate for all three systems decreases with increasing deposition temperature, which indicates that pyrolytic decomposition of TTIP at elevated temperature does not have a significant role in the growth rate. A possible explanation for a reduction in growth rate can be that increased thermal motion of the adsorbed molecules at higher temperatures contributes to steric hindrance of growth. ${ }^{8}$

Increase in index of refraction with temperature for all systems can be related to an increase in products from pyrolytic decomposition of the TTIP precursor ${ }^{28}$ leading to a larger content of $\mathrm{TiO}_{2}$ with increasing temperatures. Alternatively, an increased packing density of the organic ligands may occur with increased deposition temperature. However, the density of the deposited films does not increase in a similar rate that one would expect based on the suggestions mentioned. The density of the succinic acid based films was surprisingly high when compared to the other systems and also with anatase $\mathrm{TiO}_{2}$ $\left(3.78 \mathrm{~g} \mathrm{~cm}^{-3}\right)$. We have been unable to explain the origin for this, but have verified that the films do contain carboxylic acids by FTIR and XPS, Fig. 5 . In addition, this is also the film with the lowest index of refraction.

The succinic acid has earlier been used in MLD growth together with TMA (trimethyl aluminium) as precursor pair. This system also shows a reduction in growth rate with increasing temperature, although in two steps, a higher growth 
rate ca. $0.6 \mathrm{~nm}$ per cycle until $200{ }^{\circ} \mathrm{C}$ and a lower rate $c a .0 .3 \mathrm{~nm}$ per cycle above $225{ }^{\circ} \mathrm{C}, 7^{7}$ proving on overall a notably higher growth rate than when TTIP is used. As comparison, the film density of Al-succinic acid hybrid material made by Klepper et al. was reported to be below $2.0 \mathrm{~g} \mathrm{~cm}^{-3}$ as measured by XRR proving a distinct different nature of these films.

\section{Conclusion}

Thin films of organic-inorganic hybrid materials were successfully deposited using glycine, L-aspartic acid and, succinic acid along with TTIP. Attempts have been made using arginine as organic linker; however, it gave signs of partially decomposition during evaporation. The systems show self-limiting growth with a reduction in growth rate with increasing deposition temperatures. FTIR and XPS analysis prove a hybrid nature of the films and that the organic linkers preferably react through their carboxylic acid functional groups, while some coordination to nitrogen is possible. The films are amorphous and hydrophilic as deposited, showing a notable bioactivity. An epithelial cell, rat goblet cells can be successfully grown on these amino acid based surfaces while retaining high viability. The results confirm that the ALD/MLD technique can be used to build biocompatible substrates via molecular design and opens a field for future design of scaffolds for tissue engineering.

\section{Acknowledgements}

We thank the strategic initiative MLS at the University of Oslo for funding. We would like to thank Tor Paaske Utheim for his support and discussions on cell growth studies. We also like to thank the Norwegian national infrastructure for X-ray scattering (RECX).

\section{Notes and references}

1 T. M. Klapötke, H. Köpf, I. C. Tornieporth-Oetting and P. S. White, Angew. Chem., Int. Ed. Engl., 1994, 33, 1518-1519.

2 M. Kobayashi, V. Petrykin, K. Tomita and M. Kakihana, J. Ceram. Soc. Jpn., 2008, 116, 578-583.

3 P. Sundberg and M. Karppinen, Beilstein J. Nanotechnol., 2014, 5, 1104-1136.

4 N. P. Dasgupta, X. Meng, J. W. Elam and A. B. F. Martinson, Acc. Chem. Res., 2015, 48, 341-348.

5 M. Leskelä and M. Ritala, Thin Solid Films, 2002, 409, 138146.

6 L. Kilpi, O. M. Ylivaara, A. Vaajoki, J. Malm, S. Sintonen, M. Tuominen, R. L. Puurunen and H. Ronkainen, J. Vac. Sci. Technol., A, 2016, 34, 01A124.
7 K. B. Klepper, O. Nilsen, P. A. Hansen and H. Fjellvag, Dalton Trans., 2011, 40, 4636-4646.

8 K. B. Klepper and O. Nilsen, Dalton Trans., 2010, 39, 11628.

9 T. Suntola and J. Antson, US Pat. 4058 430, 1977.

10 A. Sood, P. Sundberg, J. Malm and M. Karppinen, Appl. Surf. Sci., 2011, 257, 6435-6439.

11 A. A. Dameron, D. Seghete, B. B. Burton, S. D. Davidson, A. S. Cavanagh, J. A. Bertrand and S. M. George, Chem. Mater., 2008, 20, 3315-3326.

12 M. Nisula and M. Karppinen, Nano Lett., 2016, 16, 12761281.

13 A. I. Abdulagatov, R. A. Hall, J. L. Sutherland, B. H. Lee, A. S. Cavanagh and S. M. George, Chem. Mater., 2012, 24, 2854-2863.

14 M. Park, S. Oh, H. Kim, D. Jung, D. Choi and J.-S. Park, Thin Solid Films, 2013, 546, 153-156.

15 A. Tanskanen and M. Karppinen, Dalton Trans., 2015, 44, 19194-19199.

16 J. M. Lackner and W. Waldhauser, J. Adhes. Sci. Technol., 2010, 24, 925-961.

17 S. L. Bellis, Biomaterials, 2011, 32, 4205-4210.

18 P. Naddeo, L. Laino, M. La Noce, A. Piattelli, A. De Rosa, G. Iezzi, G. Laino, F. Paino, G. Papaccio and V. Tirino, Dent. Mater., 2015, 31, 235-243.

19 D. S. Ryan, K. S. Bower, R. K. Sia, M. A. Shatos, R. S. Howard, M. J. Mines, R. D. Stutzman and D. A. Dartt, J. Cataract Refractive Surg., 2016, 42, 1181-1189.

20 G. Sauerbrey, Zeitschrift für Physik, 1959, 155, 206-212.

21 R. M. Silverstein, F. X. Webster, D. J. Kiemle and D. L. Bryce, Spectrometric identification of organic compounds, John Wiley \& Sons, 2014.

22 M. Badertscher, P. Bühlmann and E. Pretsch, Structure determination of organic compounds, Springer, Berlin, Heidelberg, 2008.

23 F. Verpoort, T. Haemers, P. Roose and J.-P. Maes, Appl. Spectrosc., 1999, 53, 1528-1534.

24 P. Larkin, in Infrared and Raman Spectroscopy, Elsevier, Oxford, 2011, pp. 73-115, DOI: 10.1016/b978-0-12-3869845.10006-0.

25 K. S. Kim and N. Winograd, Chem. Phys. Lett., 1975, 31, 312317.

26 N. Jiang, H. J. Zhang, S. N. Bao, Y. G. Shen and Z. F. Zhou, Phys. B, 2004, 352, 118-126.

27 M. D. Mager, V. LaPointe and M. M. Stevens, Nat. Chem., 2011, 3, 582-589.

28 M. Reinke, Y. Kuzminykh and P. Hoffmann, J. Phys. Chem. C, 2016, 120, 4337-4344. 